\title{
A Note on Risky Targets and Effort *
}

\author{
Kit Pong Wong ${ }^{\dagger}$ \\ University of Hong Kong
}

January 2017

\begin{abstract}
This note examines the effort choice problem of a decision maker (DM) who has to meet a target. The more the DM spends on effort, the more likely the target is reached. Besides the risk of missing the target despite his effort, the DM faces additional uncertainty in that both the target and the status quo are subject to exogenous shocks that are beyond the DM's control. We consider two cases: the additive case in which the DM's effort affects solely the likelihood of achieving the target, and the multiplicative case in which the DM's effort also has direct effect on the target and the status quo. Using the theory of monotone comparative statics and risk apportionment, we derive sufficient conditions under which the DM spends more on effort when the target experiences an improvement in risk via higher-order stochastic dominance.
\end{abstract}

JEL classification: C61; D81

Keywords: Effort; Monotone comparative statics; Risk apportionment; Risky targets; Stochastic dominance

*I would like to thank Rob Kaas (the editor) and two anonymous referees for their helpful comments and suggestions. The usual disclaimer applies.

${ }^{\dagger}$ School of Economics and Finance, University of Hong Kong, Pokfulam Road, Hong Kong. Tel: +8522859-1044, Fax: +852-2548-1152, e-mail: kpwong@econ.hku.hk 


\title{
A Note on Risky Targets and Effort
}

\begin{abstract}
This note examines the effort choice problem of a decision maker (DM) who has to meet a target. The more the DM spends on effort, the more likely the target is reached. Besides the risk of missing the target despite his effort, the DM faces additional uncertainty in that both the target and the status quo are subject to exogenous shocks that are beyond the DM's control. We consider two cases: the additive case in which the DM's effort affects solely the likelihood of achieving the target, and the multiplicative case in which the DM's effort also has direct effect on the target and the status quo. Using the theory of monotone comparative statics and risk apportionment, we derive sufficient conditions under which the DM spends more on effort when the target experiences an improvement in risk via higher-order stochastic dominance.
\end{abstract}

JEL classification: C61; D81

Keywords: Effort; Monotone comparative statics; Risk apportionment; Risky targets; Stochastic dominance

\section{Introduction}

In a recent article in this Journal, Chuang et al. (2013) examine the effort choice problem of a decision maker (DM) who has a target to meet. The more the DM spends on effort, the more likely the target is reached. ${ }^{1}$ Besides the risk of missing the target despite his effort, the DM faces additional uncertainty in that both the target and the status quo are subject to exogenous shocks that are beyond the DM's control. Chuang et al. (2013) focus on the comparative statics with respect to the DM's optimal effort when there are changes in risk that influence either the target or the status quo. Crainich et al. (2016) extend their analysis to allow for different risk attitudes including not only risk aversion but also risk

\footnotetext{
${ }^{1}$ The effort choice problem of Chuang et al. (2013) is analogous to the self-protection problem of Ehrlich and Becker (1972). While effort is chosen to enhance the likelihood of a positive outcome in the former problem, it is chosen to reduce the occurrence of a negative event in the latter problem.
} 
neutrality and risk loving.

Examples of the effort choice problem considered by Chuang et al. (2013) abound. For instance, firms innovate by incurring $R \& D$ expenditures to improve their products. New products are more likely to be launched, consequently making the existing products obsolete, should firms spend more on R\&D. In the worse case scenario when innovations fail, firms still have their existing products for sales. Of course, the actual demand for either the new or existing products is not completely predictable ex ante, thereby exposing the target and the status quo to substantial uncertainty. As another example, portfolio managers are commonly required to beat some benchmark returns either explicitly or implicitly. To this end, they spend resources to construct plausible trading strategies that might generate abnormal returns. If no such trading strategies are found, portfolio managers can still invest in the benchmark portfolios. In either case, the portfolio returns are influenced by market conditions and sentiments, making both the target and the status quo risky.

The purpose of this note is twofold. First, as in Crainich et al. (2016), we derive sufficient conditions under which the DM spends more on effort when the target experiences an improvement in risk in the sense of higher-order stochastic dominance. While Chuang et al. (2013) provide necessary and sufficient conditions for this comparative statics exercise, their conditions depend on endogenously chosen variables, thereby substantially reducing their usefulness. Unlike Chuang et al. (2013) and Crainich et al. (2016), both of which rely on the first- and second-order conditions for the DM's effort choice problem, we apply the theory of monotone comparative statics (Milgrom and Shannon, 1994) and risk apportionment (Eeckhoudt and Schlesinger, 2006; Eeckhoudt et al., 2009; Chiu et al., 2012) to perform the comparative statics analysis. This approach follows that of Wang and Li (2015), which shows that precautionary effort $\grave{a}$ la Eeckhoudt et at. (2012) is another trait for prudence. ${ }^{2}$ Indeed, Nocetti (2016) offers a rather general setting with one or more decision variables to study the comparative statics of higher-degree risk changes, which is applicable to generalize

\footnotetext{
${ }^{2}$ Wong (2016) extends the analysis of Wang and Li (2015) to the case of precautionary self-insurancecum-protection.
} 
many classical theories such as precautionary saving and self-protection.

Second, we consider not only the additive case in which the DM's effort affects solely the likelihood of achieving the target (Chuang et al., 2013; Crainich et al., 2016), but also the multiplicative case in which the DM's effort has direct effect on the target and the status quo. The idea is that while the target and the status quo may be measured by means of lump-sum stochastic returns as in the additive case, they may very well be measured by means of per-dollar stochastic returns as in the multiplicative case. The examples of innovative firms and portfolio managers mentioned above fall squarely into the additive and multiplicative cases, respectively.

The rest of this note is organized as follows. Section 2 delineates a variant model of Chuang et al. (2013). Section 3 derives sufficient conditions under which the DM spends more on effort when the target becomes less risky via higher-order stochastic dominance in the additive case. Section 4 extends the analysis to the multiplicative case. The final section concludes.

\section{The model}

Consider a variant model of risky targets and effort à la Chuang et al. (2013). A decision maker $(\mathrm{DM})$ has initial wealth, $w_{0}>0$, and a continuously differentiable utility function, $u(w)$, defined over his terminal wealth, $w>0$. Let $T$ be a positive integer and define $U_{T} \equiv\left\{u(w):(-1)^{n-1} u^{(n)}(w)>0\right.$ for $\left.n=1, \ldots, T\right\}$, where $u^{(n)}(w)=\mathrm{d}^{n} u(w) / \mathrm{d} w^{n}$ denotes the $n$th derivative of $u(w)$. Hence, $U_{T}$ is the set of utility functions that satisfy risk apportionment up to order $T$ (Eeckhoudt and Schlesinger, 2006). Letting $T$ go to infinity imposes $u(w)$ to have all odd derivatives positive and all even derivatives negative. In this case, $u(w)$ is completely monotone and exhibits mixed risk aversion (Caballé and Pomansky, 1996).

The DM faces an original risky situation, $\tilde{b}$, but he prefers to face an alternative risky 
situation, $\tilde{a}$, to which we refer as his target. Both $\tilde{a}$ and $\tilde{b}$ are positive continuous random variables such that $\mathrm{E}[u(\tilde{a})]>\mathrm{E}[u(\tilde{b})]$, where $\mathrm{E}[\cdot]$ is the expectation operator. To make it more likely to reach the target, the DM can spend an amount, $e$, on effort, where the expenditure, $e$, is endogenously chosen from the compact set, $\left[0, w_{0}\right] .^{3}$ Given that $e$ has been spent, the probability of achieving the target is $p(e)$ and that of remaining at the original situation is $1-p(e)$, where $p(e)$ is a continuous function such that $0 \leq p(e) \leq 1$. We assume that the DM's effort is effective in that $p(e)$ is increasing in $e$ for all $e \in\left[0, w_{0}\right]$.

Let $F(a)$ be the cumulative distribution function $(\mathrm{CDF})$ of $\tilde{a}$ over support $[\underline{a}, \bar{a}]$, where $0 \leq \underline{a}<\bar{a}$. Define $F_{1}(a)=F(a)$ and $F_{n+1}(a)=\int_{\underline{a}}^{a} F_{n}(x) \mathrm{d} x$ for all $n=1, \ldots, N-1$ and $a \in[\underline{a}, \bar{a}]$, where $N \geq 2$. Let $\tilde{a}^{\prime}$ be another random variable that is distributed according to the CDF, $G(a)$, over support $[\underline{a}, \bar{a}]$. Define $G_{n}(a)$ in an analogous manner as $F_{n}(a)$ for $n=1$, $\ldots, N$. We say that $\tilde{a}^{\prime}$ dominates $\tilde{a}$ via $N$ th-order stochastic dominance if $G_{n}(\bar{a}) \leq F_{n}(\bar{a})$ for all $n=1, \ldots, N$, and $G_{N}(a) \leq F_{N}(a)$ for all $a \in[\underline{a}, \bar{a}]$, where the inequality is strict for some $a$. In the special case wherein $G_{n}(\bar{a})=F_{n}(\bar{a})$ for all $n=1, \ldots, N$, the first $N-1$ moments of the two distributions coincide. In this case, we say that $\tilde{a}$ has more $N$ th-degree risk than $\tilde{a}^{\prime}$ in the sense of Ekern (1980).

\section{Additive risky targets and effort}

As in Chuang et al. (2013), we consider in this section the case that the risky target and status quo are additive in nature so that the DM's expected utility is given by

$$
f(e)=p(e) \mathrm{E}\left[u\left(w_{0}-e+\tilde{a}\right)\right]+[1-p(e)] \mathrm{E}\left[u\left(w_{0}-e+\tilde{b}\right)\right],
$$

The DM's ex-ante decision problem is to choose $e \in\left[0, w_{0}\right]$ so as to maximize $f(e)$. Since $f(e)$ is a continuous function of $e$, the set, $\arg \max _{e \in\left[0, w_{0}\right]} f(e)$, is non-empty, and plausibly not a singleton. Let $e_{1}^{*}$ be an element in $\arg \max _{e \in\left[0, w_{0}\right]} f(e)$.

\footnotetext{
${ }^{3}$ An alternative way is to specify the effort cost as disutility (see Chuang et al., 2013). In this case, it is easily verified that the results of Propositions 1 and 2 remain intact.
} 
Following Chuang et al. (2013), we conduct the comparative statics with respect to the DM's optimal effort when the risky target, $\tilde{a}$, changes to $\tilde{a}^{\prime}$, where $\tilde{a}^{\prime}$ dominates $\tilde{a}$ via $N$ th-order stochastic dominance and $N \geq 1$. In this case, the DM's expected utility becomes

$$
g(e)=p(e) \mathrm{E}\left[u\left(w_{0}-e+\tilde{a}^{\prime}\right)\right]+[1-p(e)] \mathrm{E}\left[u\left(w_{0}-e+\tilde{b}\right)\right]
$$

The DM's ex-ante decision problem is to choose $e \in\left[0, w_{0}\right]$ so as to maximize $g(e)$. Since $g(e)$ is a continuous function of $e$, the set, $\arg \max _{e \in\left[0, w_{0}\right]} g(e)$, is non-empty, and plausibly not a singleton. Let $e_{2}^{*}$ be an element in $\arg \max _{e \in\left[0, w_{0}\right]} g(e)$.

To examine the DM's optimal effort when the target becomes less risky, we have to compare the two sets, $\arg \max _{e \in\left[0, w_{0}\right]} f(e)$ and $\arg \max _{e \in\left[0, w_{0}\right]} g(e)$. This falls into a principal concern in the theory of monotone comparative statics (Milgrom and Shannon, 1994). The following well-known theorem is adapted from Topkis $(1978,1998)$.

Topkis' Monotonicity Theorem. If the function, $\phi(e, \theta)$, satisfies the single-crossing condition in $(e, \theta)$, i.e.,

$$
\phi\left(e_{2}, \theta_{1}\right)-\phi\left(e_{1}, \theta_{1}\right) \geq 0 \Rightarrow \phi\left(e_{2}, \theta_{2}\right)-\phi\left(e_{1}, \theta_{2}\right)>0
$$

for all $e_{2}>e_{1}$ and $\theta_{2}>\theta_{1}$, then $e_{\theta_{2}}^{*} \geq e_{\theta_{1}}^{*}$ for all $\theta_{2}>\theta_{1}$, where $e_{\theta}^{*} \in \arg \max _{e \in\left[0, w_{0}\right]} \phi(e, \theta)$.

Proof. Since $e_{\theta}^{*} \in \arg \max _{e \in\left[0, w_{0}\right]} \phi(e, \theta)$, we must have $\phi\left(e_{\theta_{1}}^{*}, \theta_{1}\right)-\phi\left(e_{\theta_{2}}^{*}, \theta_{1}\right) \geq 0$. Suppose that $e_{\theta_{1}}^{*}>e_{\theta_{2}}^{*}$, where $\theta_{2}>\theta_{1}$. It then follows from the single-crossing condition (3) that $\phi\left(e_{\theta_{1}}^{*}, \theta_{2}\right)-\phi\left(e_{\theta_{2}}^{*}, \theta_{2}\right)>0$, which is contradictory to $e_{\theta_{2}}^{*} \in \arg \max _{e \in\left[0, w_{0}\right]} \phi\left(e, \theta_{2}\right)$. Hence, it must be true that $e_{\theta_{2}}^{*} \geq e_{\theta_{1}}^{*}$ for all $\theta_{2}>\theta_{1}$.

In the following proposition, we derive sufficient, but not necessary, conditions under which the DM spends more on effort when the target becomes less risky, i.e., $e_{2}^{*} \geq e_{1}^{*}$. 
Proposition 1. Given that the risky situations are additive in nature, and that the risky target, $\tilde{a}$, experiences an improvement in risk to $\tilde{a}^{\prime}$ via $N$ th-order stochastic dominance, the DM spends more on effort, i.e., $e_{2}^{*} \geq e_{1}^{*}$, if the DM's utility function satisfies that $u(w) \in U_{N+1}$.

Proof. Define $f(e)=\phi(e, 1)$ and $g(e)=\phi(e, 2)$. For all $e_{2}>e_{1}$, it follows from Eq. (1) that $f\left(e_{2}\right)-f\left(e_{1}\right) \geq 0$ is equivalent to

$$
\begin{aligned}
& p\left(e_{2}\right) \mathrm{E}\left[u\left(w_{0}-e_{2}+\tilde{a}\right)\right]-p\left(e_{1}\right) \mathrm{E}\left[u\left(w_{0}-e_{1}+\tilde{a}\right)\right] \\
& \geq\left[1-p\left(e_{1}\right)\right] \mathrm{E}\left[u\left(w_{0}-e_{1}+\tilde{b}\right)\right]-\left[1-p\left(e_{2}\right)\right] \mathrm{E}\left[u\left(w_{0}-e_{2}+\tilde{b}\right)\right] .
\end{aligned}
$$

Since $\tilde{a}^{\prime}$ dominates $\tilde{a}$ via $N$ th-order stochastic dominance and $w_{0}-e_{1}$ dominates $w_{0}-e_{2}$ via first-order stochastic dominance, Theorem 3 of Eeckhoudt et al. (2009) implies that the 50-50 binary lottery, $\left[w_{0}-e_{2}+\tilde{a}^{\prime} ; w_{0}-e_{1}+\tilde{a}\right]$, dominates the 50-50 binary lottery, $\left[w_{0}-e_{1}+\tilde{a}^{\prime} ; w_{0}-e_{2}+\tilde{a}\right]$, via $(N+1)$ th-order stochastic dominance. Since $u(w) \in U_{N+1}$, it follows from the $(N+1)$ th-order stochastic dominance that

$$
\frac{1}{2} \mathrm{E}\left[u\left(w_{0}-e_{2}+\tilde{a}^{\prime}\right)\right]+\frac{1}{2} \mathrm{E}\left[u\left(w_{0}-e_{1}+\tilde{a}\right)\right]>\frac{1}{2} \mathrm{E}\left[u\left(w_{0}-e_{1}+\tilde{a}^{\prime}\right)\right]+\frac{1}{2} \mathrm{E}\left[u\left(w_{0}-e_{2}+\tilde{a}\right)\right] .
$$

Rearranging terms, we can write inequality (5) as

$$
\mathrm{E}\left[u\left(w_{0}-e_{2}+\tilde{a}^{\prime}\right)\right]-\mathrm{E}\left[u\left(w_{0}-e_{2}+\tilde{a}\right)\right]>\mathrm{E}\left[u\left(w_{0}-e_{1}+\tilde{a}^{\prime}\right)\right]-\mathrm{E}\left[u\left(w_{0}-e_{1}+\tilde{a}\right)\right] .
$$

Since $\tilde{a}^{\prime}$ dominates $\tilde{a}$ via $N$ th-order stochastic dominance and $u(w) \in U_{N+1} \subset U_{N}$, we have $\mathrm{E}\left[u\left(\tilde{a}^{\prime}\right)\right]>\mathrm{E}[u(\tilde{a})]$. Since $p\left(e_{2}\right)>p\left(e_{1}\right)$, we multiply $p\left(e_{2}\right)$ to the left-hand side of inequality (6) and $p\left(e_{1}\right)$ to the right-hand side of inequality (6) to yield

$$
\begin{aligned}
& p\left(e_{2}\right) \mathrm{E}\left[u\left(w_{0}-e_{2}+\tilde{a}^{\prime}\right)\right]-p\left(e_{1}\right) \mathrm{E}\left[u\left(w_{0}-e_{1}+\tilde{a}^{\prime}\right)\right] \\
& >p\left(e_{2}\right) \mathrm{E}\left[u\left(w_{0}-e_{2}+\tilde{a}\right)\right]-p\left(e_{1}\right) \mathrm{E}\left[u\left(w_{0}-e_{1}+\tilde{a}\right)\right]
\end{aligned}
$$




$$
\geq\left[1-p\left(e_{1}\right)\right] \mathrm{E}\left[u\left(w_{0}-e_{1}+\tilde{b}\right)\right]-\left[1-p\left(e_{2}\right)\right] \mathrm{E}\left[u\left(w_{0}-e_{2}+\tilde{b}\right)\right]
$$

where the last inequality follows from inequality (4). It follows from Eq. (2) that inequality (7) is equivalent to $g\left(e_{2}\right)-g\left(e_{1}\right)>0$. Hence, the single-crossing condition (3) is satisfied for $\theta_{1}=1$ and $\theta_{2}=2$. It then follows from Topkis' Monotonicity Theorem that $e_{2}^{*} \geq e_{1}^{*}$.

When $\tilde{a}$ has more $N$ th-degree risk than $\tilde{a}^{\prime}$ in the sense of Ekern (1980), it is straightforward to verify that $e_{2}^{*} \geq e_{1}^{*}$ if $(-1)^{N-1} u^{(N)}(w)>0$ and $(-1)^{N} u^{(N+1)}(w)>0$. Indeed, Crainich et al. (2016) derive the same sufficient conditions using the traditional approach that relies on the first- and second-order conditions for the DM's decision problem. ${ }^{4} \mathrm{We}$ count on the alternative approach adopted from the theory of monotone comparative statics and risk apportionment. Proposition 1 shows that the sole driver is the single-crossing condition (3) and not the first- and second-order conditions for the DM's decision problem, making the comparative statics results completely general.

Using the traditional approach, Chuang et al. (2013) derive necessary and sufficient condition under which $e_{2}^{*} \geq e_{1}^{*}$ when $\tilde{a}$ has more $N$ th-degree risk than $\tilde{a}^{\prime}$ in the sense of Ekern (1980). Specifically, using our notation, we can state the condition of Chuang et al. (2013) as follows:

$$
-\frac{u^{(N+1)}\left(w_{0}-e_{1}^{*}+a\right)}{u^{(N)}\left(w_{0}-e_{1}^{*}+a\right)}>-\frac{p^{\prime}\left(e_{1}^{*}\right)}{p\left(e_{1}^{*}\right)},
$$

for all $a \in[\underline{a}, \bar{a}]$. Given that $(-1)^{N-1} u^{(N)}(w)>0$ and $(-1)^{N} u^{(N+1)}(w)>0$, the lefthand side of condition (8) becomes the $N$ th-degree Arrow-Pratt measure of absolute risk aversion (Caballé and Pomansky, 1996; Jindapon and Neilson, 2007), which is positive. Hence, condition (8) holds and $e_{2}^{*} \geq e_{1}^{*}$. On the other hand, even when $u^{(N)}(w)>0$ and $u^{(N+1)}(w)>0$, condition (8) may still hold provided that the left-hand side is less negative relative to the right-hand side, thereby rendering $e_{2}^{*} \geq e_{1}^{*}$. As such, $u(w) \in U_{N+1}$ is sufficient but not necessary for $e_{2}^{*} \geq e_{1}^{*}$ when $\tilde{a}$ experiences an improvement in risk to $\tilde{a}^{\prime}$

\footnotetext{
${ }^{4}$ It is well-known from the literature on effort that the second-order conditions for effort-choice problems need not hold. See also Crainich et al. (2016).
} 
via $N$ th-order stochastic dominance. It is evident from condition (8) that it depends on $e_{1}^{*}$, which is endogenously determined, thereby making condition (8) far less informative.

To see the underlying intuition for Proposition 1, we follow Eeckhoudt and Schlesinger (2006) to define the utility premium for shifting the risk from $\tilde{a}^{\prime}$ to $\tilde{a}$, where $\tilde{a}^{\prime}$ dominates $\tilde{a}$ via $N$ th-order stochastic dominance, as

$$
\pi(e)=p(e) \mathrm{E}\left[u\left(w_{0}-e+\tilde{a}\right)\right]-p(e) \mathrm{E}\left[u\left(w_{0}-e+\tilde{a}^{\prime}\right)\right]
$$

The utility premium, $\pi(e)$, which is negative if, and only if, the DM's utility function satisfies that $u(w) \in U_{N}$. As such, $\pi(e)$ measures the "pain" caused by the increase in risk from $\tilde{a}^{\prime}$ to $\tilde{a}$ in terms of the loss of expected utility. Differentiating Eq. (9) with respect to $e$ yields

$$
\begin{aligned}
\pi^{\prime}(e)= & p^{\prime}(e)\left\{\mathrm{E}\left[u\left(w_{0}-e+\tilde{a}\right)\right]-\mathrm{E}\left[u\left(w_{0}-e+\tilde{a}^{\prime}\right]\right\}\right. \\
& -p(e)\left\{\mathrm{E}\left[u^{\prime}\left(w_{0}-e+\tilde{a}\right)\right]-\mathrm{E}\left[u^{\prime}\left(w_{0}-e+\tilde{a}^{\prime}\right)\right]\right\}
\end{aligned}
$$

The expression inside the curly brackets of the first term on the right-hand side of Eq. (10) is positive if, and only if, $u(w) \in U_{N}$. The expression inside the curly brackets of the second term on the right-hand side of Eq. (10) is negative if, and only if, $u(w) \in U_{N+1}$. Since $p^{\prime}(e)>0$, we conclude that $\pi^{\prime}(e)<0$ if $u(w) \in U_{N+1}$. The DM as such has less incentives to spend on effort that leads him to the more risky target, thereby rendering that $e_{1}^{*} \leq e_{2}^{*}$.

\section{Multiplicative risky targets and effort}

In this section, we consider the case that the risky target and status quo are multiplicative in nature so that the DM's expected utility is given by

$$
f(e)=p(e) \mathrm{E}\left[u\left(\left(w_{0}-e\right) \tilde{a}\right)\right]+[1-p(e)] \mathrm{E}\left[u\left(\left(w_{0}-e\right) \tilde{b}\right)\right]
$$


Let $e_{1}^{*}$ be an element in $\arg \max _{e \in\left[0, w_{0}\right]} f(e)$. When the risky target, $\tilde{a}$, changes to $\tilde{a}^{\prime}$, where $\tilde{a}^{\prime}$ dominates $\tilde{a}$ via $N$ th-order stochastic dominance and $N \geq 1$, the DM's expected utility becomes

$$
g(e)=p(e) \mathrm{E}\left[u\left(\left(w_{0}-e\right) \tilde{a}^{\prime}\right)\right]+[1-p(e)] \mathrm{E}\left[u\left(\left(w_{0}-e\right) \tilde{b}\right)\right]
$$

Let $e_{2}^{*}$ be an element in arg $\max _{e \in\left[0, w_{0}\right]} g(e)$.

Denote $R^{(n)}(w)=-w u^{(n+1)}(w) / u^{(n)}(w)$ as the $n$ th-degree Arrow-Pratt measure of relative risk aversion. In the following proposition, we derive sufficient, but not necessary, conditions under which the DM spends more on effort when the target becomes less risky, i.e., $e_{2}^{*} \geq e_{1}^{*}$.

Proposition 2. Given that the risky situations are multiplicative in nature, and that the risky target, $\tilde{a}$, experiences an improvement in risk to $\tilde{a}^{\prime}$ via $N$ th-order stochastic dominance, the DM spends more on effort, i.e., $e_{2}^{*} \geq e_{1}^{*}$, if the DM's utility function satisfies that $u(w) \in U_{N+1}$ and $R^{(n)}(w) \geq n$ for $n=1, \ldots, N$.

Proof. Define $f(e)=\phi(e, 1)$ and $g(e)=\phi(e, 2)$. For all $e_{2}>e_{1}$, it follows from Eq. (11) that $f\left(e_{2}\right)-f\left(e_{1}\right) \geq 0$ is equivalent to

$$
\begin{aligned}
& p\left(e_{2}\right) \mathrm{E}\left[u\left(\left(w_{0}-e_{2}\right) \tilde{a}\right)\right]-p\left(e_{1}\right) \mathrm{E}\left\{u\left[\left(w_{0}-e_{1}\right) \tilde{a}\right]\right\} \\
& \geq\left[1-p\left(e_{1}\right)\right] \mathrm{E}\left[u\left(\left(w_{0}-e_{1}\right) \tilde{b}\right)\right]-\left[1-p\left(e_{2}\right)\right] \mathrm{E}\left[u\left(\left(w_{0}-e_{2}\right) \tilde{b}\right)\right] .
\end{aligned}
$$

Since $\tilde{a}^{\prime}$ dominates $\tilde{a}$ via $N$ th-order stochastic dominance and $w_{0}-e_{1}$ dominates $w_{0}-e_{2}$ via first-order stochastic dominance, Theorem 3 of Chiu et al. (2012) implies that the DM prefers the 50-50 binary lottery, $\left[\left(w_{0}-e_{2}\right) \tilde{a}^{\prime} ;\left(w_{0}-e_{1}\right) \tilde{a}\right]$, to the 50-50 binary lottery, $\left[\left(w_{0}-e_{1}\right) \tilde{a}^{\prime} ;\left(w_{0}-e_{2}\right) \tilde{a}\right]$, if $u(x) \in U_{N+1}$ and $R^{(n)}(w) \geq n$ for $n=1, \ldots, N$ so that

$$
\frac{1}{2} \mathrm{E}\left[u\left(\left(w_{0}-e_{2}\right) \tilde{a}^{\prime}\right)\right]+\frac{1}{2} \mathrm{E}\left[u\left(\left(w_{0}-e_{1}\right) \tilde{a}\right)\right]
$$




$$
>\frac{1}{2} \mathrm{E}\left[u\left(\left(w_{0}-e_{1}\right) \tilde{a}^{\prime}\right)\right]+\frac{1}{2} \mathrm{E}\left[u\left(\left(w_{0}-e_{2}\right) \tilde{a}\right)\right] .
$$

Rearranging terms, we can write inequality (14) as

$$
\mathrm{E}\left[u\left(\left(w_{0}-e_{2}\right) \tilde{a}^{\prime}\right)\right]-\mathrm{E}\left[u\left(\left(w_{0}-e_{2}\right) \tilde{a}\right)\right]>\mathrm{E}\left[u\left(\left(w_{0}-e_{1}\right) \tilde{a}^{\prime}\right)\right]-\mathrm{E}\left[u\left(\left(w_{0}-e_{1}\right) \tilde{a}\right)\right] .
$$

Since $\tilde{a}^{\prime}$ dominates $\tilde{a}$ via $N$ th-order stochastic dominance and $u(w) \in U_{N+1} \subset U_{N}$, we have $\mathrm{E}\left[u\left(\tilde{a}^{\prime}\right)\right]>\mathrm{E}[u(\tilde{a})]$. Since $p\left(e_{2}\right)>p\left(e_{1}\right)$, we multiply $p\left(e_{2}\right)$ to the left-hand side of inequality (15) and $p\left(e_{1}\right)$ to the right-hand side of inequality (15) to yield

$$
\begin{aligned}
& p\left(e_{2}\right) \mathrm{E}\left[u\left(\left(w_{0}-e_{2}\right) \tilde{a}^{\prime}\right)\right]-p\left(e_{1}\right) \mathrm{E}\left[u\left(\left(w_{0}-e_{1}\right) \tilde{a}^{\prime}\right)\right] \\
& >p\left(e_{2}\right) \mathrm{E}\left[u\left(\left(w_{0}-e_{2}\right) \tilde{a}\right)\right]-p\left(e_{1}\right) \mathrm{E}\left[u\left(\left(w_{0}-e_{1}\right) \tilde{a}\right)\right] \\
& \geq\left[1-p\left(e_{1}\right)\right] \mathrm{E}\left[u\left(\left(w_{0}-e_{1}\right) \tilde{b}\right)\right]-\left[1-p\left(e_{2}\right)\right] \mathrm{E}\left[u\left(\left(w_{0}-e_{2}\right) \tilde{b}\right)\right],
\end{aligned}
$$

where the last inequality follows from inequality (13). It follows from Eq. (12) that inequality (16) is equivalent to $g\left(e_{2}\right)-g\left(e_{1}\right)>0$. Hence, the single-crossing condition (3) is satisfied for $\theta_{1}=1$ and $\theta_{2}=2$. It then follows from Topkis' Monotonicity Theorem that $e_{2}^{*} \geq e_{1}^{*}$.

When $\tilde{a}$ has more $N$ th-degree risk than $\tilde{a}^{\prime}$ in the sense of Ekern (1980), it is easily verified that $e_{2}^{*} \geq e_{1}^{*}$ if $(-1)^{N-1} u^{(N)}(w)>0,(-1)^{N} u^{(N+1)}(w)>0$, and $R^{(N)}(w) \geq N$. To see the underlying intuition for Proposition 2, we follow Eeckhoudt and Schlesinger (2006) to define the utility premium for shifting the risk from $\tilde{a}^{\prime}$ to $\tilde{a}$, where $\tilde{a}^{\prime}$ dominates $\tilde{a}$ via $N$ th-order stochastic dominance, as

$$
\pi(e)=p(e) \mathrm{E}\left[u\left(\left(w_{0}-e\right) \tilde{a}\right)\right]-p(e) \mathrm{E}\left[u\left(\left(w_{0}-e\right) \tilde{a}^{\prime}\right)\right]
$$

The utility premium, $\pi(e)$, which is negative if, and only if, the DM's utility function satisfies that $u(w) \in U_{N}$. As such, $\pi(e)$ measures the "pain" caused by the increase in risk 
from $\tilde{a}^{\prime}$ to $\tilde{a}$ in terms of the loss of expected utility. Differentiating Eq. (17) with respect to $e$ yields

$$
\begin{aligned}
\pi^{\prime}(e)= & p^{\prime}(e)\left\{\mathrm{E}\left[u\left(\left(w_{0}-e\right) \tilde{a}\right)\right]-\mathrm{E}\left[u\left(\left(w_{0}-e\right) \tilde{a}^{\prime}\right)\right]\right\} \\
& -p(e)\left\{\mathrm{E}\left[u^{\prime}\left(\left(w_{0}-e\right) \tilde{a}\right) \tilde{a}\right]-\mathrm{E}\left[u^{\prime}\left(\left(w_{0}-e\right) \tilde{a}^{\prime}\right) \tilde{a}^{\prime}\right]\right\}
\end{aligned}
$$

The expression inside the curly brackets of the first term on the right-hand side of Eq. (18) is positive if, and only if, $u(w) \in U_{N}$. According to Theorem 3 of Chiu et al. (2012), the expression inside the curly brackets of the second term on the right-hand side of Eq. (18) is negative if $u(w) \in U_{N+1}$ and $R^{(n)}(w) \geq n$ for $n=1, \ldots, N$. Since $p^{\prime}(e)>0$, we conclude

that $\pi^{\prime}(e)<0$ if $u(w) \in U_{N+1}$ and $R^{(n)}(w) \geq n$ for $n=1, \ldots, N$. The DM as such has less incentives to spend on effort that leads him to the more risky target, thereby rendering that $e_{1}^{*} \leq e_{2}^{*}$.

\section{Conclusion}

In this note, we revisit the effort choice problem of a decision maker (DM) who has to meet a target, as developed by Chuang et al. (2013). The more the DM spends on effort, the more likely the target is reached. Besides the risk of missing the target despite his effort, the DM faces additional uncertainty in that both the target and the status quo are subject to exogenous shocks that are beyond the DM's control. We consider two cases: the additive case in which the DM's effort affects solely the likelihood of achieving the target, and the multiplicative case in which the DM's effort also has direct effect on the target and the status quo. Using the theory of monotone comparative statics (Milgrom and Shannon, 1994) and risk apportionment (Eeckhoudt and Schlesinger, 2006; Eeckhoudt et al., 2009; Chiu et al., 2012), we derive sufficient conditions under which the DM spends more on effort when the target experiences an improvement in risk via higher-order stochastic dominance. 


\section{References}

Caballé, J., Pomansky, A., 1996. Mixed risk aversion. Journal of Economic Theory 71, $485-513$.

Chiu, W.H., Eeckhoudt, L., Rey, B., 2012. On relative and partial risk attitudes: Theory and implications. Economic Theory 50, 151-167.

Chuang, O-C., Eeckhoudt, L., Huang, R.J., Tzeng, L.Y., 2013. Risky targets and effort. Insurance: Mathematics and Economics 52, 465-468.

Crainich, D., Eeckhoudt, L., Menegatti, M., 2016. Changing risks and optimal effort. Journal of Economic Behavior and Organization 125, 97-106.

Eeckhoudt, L., Huang, R.J., Tzeng, L.Y., 2012. Precautionary effort: A new look. Journal of Risk and Insurance 79, 585-590.

Eeckhoudt, L., Schlesinger, H., 2006. Putting risk in its proper place. American Economic Review 96, 280-289.

Eeckhoudt, L., Schlesinger, H., Tsetlin, I., 2009. Apportioning of risks via stochastic dominance. Journal of Economic Theory 144, 994-1003.

Ehrlich, I., Becker, G.S., 1972. Market insurance, self-insurance, and self-protection. Journal of Political Economy 80, 623-648.

Ekern, S., 1980. Increasing $N$ th degree risk. Economics Letters 6, 329-333.

Jindapon, P., Neilson, W.S., 2007. Higher-order generalizations of Arrow-Pratt and Ross risk aversion: A comparative statics approach. Journal of Economic Theory 136, 719728.

Milgrom, P., Shannon, C., 1994. Monotone comparative statics. Econometrica 62, 157-180.

Nocetti, D. C., 2016. Robust comparative statics of risk changes. Management Science 62, $1381-1392$. 
Topkis, D.M., 1978. Minimizing a submodular function on a lattice. Operations Research 26, 305-321.

Topkis, D.M., 1998. Supermodularity and Complementarity. Princeton University Press, Princeton, New Jersey.

Wang, J., Li, J., 2015. Precautionary effort: Another trait for prudence. Journal of Risk and Insurance 82, 977-983.

Wong, K.P., 2016. Precautionary self-insurance-cum-protection. Economics Letters 145, 152-156. 